\title{
TTR
}

Traduction, terminologie, re?daction

\section{Cay Dollerup \& Anne Loddegaard, eds. Teaching Translation and Interpreting. Training, Talent and Experience. Amsterdam/Philadelphia, John Benjamins, 1992, 343 p.}

\section{Jean Delisle}

Volume 6, numéro 2, 2e semestre 1993

Traduction, mixité, politique

URI : https://id.erudit.org/iderudit/037159ar

DOI : https://doi.org/10.7202/037159ar

Aller au sommaire du numéro

Éditeur(s)

Association canadienne de traductologie

ISSN

0835-8443 (imprimé)

1708-2188 (numérique)

Découvrir la revue

Citer ce compte rendu

Delisle, J. (1993). Compte rendu de [Cay Dollerup \& Anne Loddegaard, eds. Teaching Translation and Interpreting. Training, Talent and Experience. Amsterdam/Philadelphia, John Benjamins, 1992, 343 p.] TTR, 6(2), 178-181. https://doi.org/10.7202/037159ar

Tous droits réservés @ C TTR: traduction, terminologie, rédaction — Les auteurs, 1993 
André Lefevere University of Texas at Austin

\section{Cay Dollerup \& Anne Loddegaard, eds. Teaching Translation and Interpreting. Training, Talent and Experience. Amsterdam/Philadelphia, John Benjamins, 1992, 343 p.}

Cet ouvrage réunit les trente-cinq communications présentées à Elsinore (Danemark) lors du premier congrès organisé sous les auspices de Language International, dont Geoffrey Kingscott est le rédacteur en chef, et de la maison d'édition John Benjamins (Pays- 
Bas). Ce congrès eut lieu du 31 mai au 2 juin 1991. C'est donc un véritable tour de force qu'ont réalisé les deux directeurs de cet ouvrage en publiant ce recueil de textes dans l'année qui a suivi le congrès. Mais il faut les féliciter aussi et surtout pour l'exceptionnelle qualité de leur travail d'édition. Chacun sait que préparer les actes d'un congrès en vue de leur publication est une tâche ingrate, mais dans ce cas-ci, les responsables ont pris d'heureuses initiatives.

En effet, dans un souci fort louable de clarté et de lisibilité, ils ont extirpé "mercilessly» (p. 1) presque tout le jargon et tous les acronymes des communications. Il faut leur savoir gré d'avoir ainsi produit un ouvrage d'une grande clarté et qui se caractérise par son unité de style. Il s'en dégage une étonnante homogénéité; on a l'impression qu'il est l'œuvre d'une même plume. Ils n'ont pas opéré un choix parmi les communications présentées, mais toutes méritaient $\mathrm{d}$ 'être publiées. Aucune ne "réinvente la roue», même si certains auteurs apportent une contribution plus originale que d'autres. On peut même dire que, dans l'ensemble, cet ouvrage trace un bilan fidèle et assez complet de la situation actuelle de l'enseignement de la traduction et de l'interprétation dans le monde, en Occident tout au moins. Bien que toutes les communications soient rédigées en anglais ("English is the language of exchange of ideas, not of power and oppression", précisent les directeurs en guise d'excuse), un large éventail de pays sont représentés: Danemark, Royaume-Uni, Israël, Allemagne, Espagne, France, Autriche, Canada, Finlande, mais aussi des pays dont on connait moins la situation de l'enseignement de la traduction comme l'Iran, le sultanat de Brunei, l'Afrique du Sud, le Chili, l'Uruguay et la Bulgarie. Par ailleurs, les directeurs ont eu la bonne idée de regrouper en fin de volume tous les ouvrages cités, ce qui allège considérablement la présentation. Enfin, chose rarissime dans des actes de congrès, ils ont ajouté un index des sujets traités ce qui transforme ce recueil de textes en ouvrage de référence facile à consulter. Détaillé et très bien fait, cet index de douze pages décuple l'utilité de la publication. Vraiment, il faut le répéter, Cay Dollerup et Anne Loddegaard ont accompli un travail remarquable dans un temps record. $D^{\prime}$ une présentation impeccable, cet ouvrage est un modèle du genre, un exemple à suivre.

Teaching Translation and Interpreting est un heureux mélange de considérations théoriques et pratiques sur l'art (ou la technique) 
de l'enseignement de ces deux professions-sœurs. Tous les principaux aspects de cette problématique y sont abordés: méthodes d'enseignement, composition des programmes, apport des autres disciplines, intégration des nouveaux diplômés au marché du travail, évaluation des apprentissages, rôle de la théorie, etc. Il est impossible de résumer le contenu de chacune des communications, dont plusieurs présentent des points de vue originaux. À la lecture de cet ouvrage, on note certaines lignes de force, certaines idées qui reviennent comme des leitmotive sous la plume des auteurs, pourtant venus d'horizons très différents. Plusieurs exposés réaffirment la nécessité de dispenser un enseignement sui generis de la traduction professionnelle, distinct de la formation donnée en linguistique ou en langues étrangères, ce qui amène ces auteurs, dont Mary SNELL-HORNBY, à préciser la nature exacte de la traduction professionnelle: «Thus translation is primarily a sociocultural activity which presupposes not only language competence but also extensive factual and encyclopaedic knowledge as well as familiarity with the everyday norms and conventions of both source and target culture" (p. 11). Et plus loin elle ajoute: "This culturally based theoretical approach $[. .$.$] has enabled us [Translation and Interpreting Institute$ (Vienne)] to emancipate our concept of translation from the constraints of the linguistic approach and to view our subject as an interdisciplinary field of study in its own right» (pp. 21-22). Pour sa part, Christiane NORD, dans un excellent article qui résume l'optique de son livre Text Analysis in Translation (1991)' énumère les compétences propres du traducteur, et celles-ci sont loin de se limiter à une simple compétence linguistique.

Trois tendances de fond se dégagent de la trentaine de communications présentées: 1) le rôle et l'utilité de la théorie dans les programmes de formation; 2) le désir des pédagogues de structurer leur enseignement de façon plus systématique; 3) la place grandissante qu'occupe l'analyse du discours en théorie comme en pédagogie de la traduction. Voyons brièvement chacun de ces trois points.

1.

Voir plus bas le compte rendu de cet ouvrage par Anthony Pym (NDLR). 
1. Éternel sujet de controverse, l'utilité de la théorie dans les programmes de formation semble encore poser problème. Les praticiens et les pédagogues ne voient pas les choses du même œil, sans parler des étudiants eux-mêmes qui, universellement, semblent avoir une aversion innée pour les considérations d'ordre théorique. Les étudiants du niveau du baccalauréat tout au moins. "And there still exists a barrier of suspicion between theorists and practising translators» (Gerard MCALESTER, p. 293). L'arrimage de la théorie et de la pratique n'est pas facile: "How does one strike the right balance between theory and practice?", se demande Giovanni PONTIERO (p. 199). Si, aux yeux des étudiants, on peut apprendre à traduire sans connaître la théorie, pour leurs professeurs, il est absolument essentiel de procéder à une réflexion théorique dans le cadre d'une formation de niveau universitaire, car, comme le dit fort judicieusement Brian MOSSOP, "we do not want to be preparing unthinking language engineers or human word processors" (p. 88). Un des rôles de la théorie, selon ce pédagogue, est de fournir le métalangage de la discipline: «Students need to learn a vocabulary for talking about translation problems" (p. 84). Pour sa part, l'interprète Ingrid KURZ rappelle que «any sound pedagogical approach requires more than a 'gut feeling'» (p. 247).

En outre, la théorie se révèle utile en traduction comme en interprétation non seulement pour comprendre le processus de cette opération mentale complexe, mais aussi pour fournir un fondement raisonné aux exercices pratiques d'apprentissage. Elle est indispensable également en docimologie: "Critics often judge translations in terms of personal taste, rather than of concrete criteria. But this subjective approach cannot be used by a teacher of translation who has to evaluate and score students' work on the basis of concrete criteria during a course and at the finals" (p. 271), écrit avec beaucoup d'à propos Farzaneh FARAHZAD dans un bon article «Testing Achievement in Translation Classes». Anthony PYM abonde dans le même sens: "The identification and analysis of translation errors requires a strong conceptual framework before it can ensure any heuristic validity" (p. 279). Juan C. SAGER considère lui aussi «that an understanding of terminology requires a minimum of theory» (p. 113). Selon Rune INGO, «translation theory is important because it offers us a chance to rise above grass-root level, to see the wood and not just the trees, to understand how phenomena are connected, to establish principles [...]» (p. 49). Maria 
Antonia ALVAREZ exprime un point de vue tout à fait identique dans sa communication (p. 151). En somme, pour reprendre la formule de Sergio VIAGGIO citée par Franz PÖCHHACKER «translatology, like any science, should be 'experience made awareness'» (p. 220). Mais Margaret F. LANG pose la condition suivante: "Success of any theoretical component in vocational training is judged, by student and teachers, by its relevance and applicability to the skill being acquired" (p. 207). Et elle ajoute à la page suivante: «It would be regrettable if the only reason to acquire a theory of translating were to bestow on the discipline some sort of respectability. Rather, the theory should be looked on as providing a conceptual basis for practical application in the classroom and in professional life" (p. 208). Jamais on n'aura autant parlé de théorie dans un ouvrage traitant de pédagogie de la traduction. N'est-ce pas au fond parce que l'un et l'autre sont indissociables?

2. Le désir des pédagogues d'organiser leur enseignement pratique de façon systématique ressort de bon nombre de communications. "I realized, confie Patricia HÖRMANN, it was not university-like to have students confronted with only translation exercises in a first course of translation" (p. 59). Pour organiser l'enseignement de façon méthodique et progressive, Christiane NORD propose de s'élever au-dessus des difficultés particulières propres à un texte donné pour traiter plutôt des grandes catégories de problemes: "In translator training, we are not interested in the individual translation problem arising in a particular translation task but in categories of translation problems which can be expected to arise in other translation tasks as well» (p. 45). La présentation systématique des difficultés récurrentes de traduction s'accompagne aussi d'un réel souci de la part des pédagogues de varier la présentation de la matière en salle de classe afin d'optimaliser l'efficacité de l'enseignement. Maria Julia SAINZ présente à cet égard plusieurs techniques «to depart from the classic Teacher-Student ping-pong class» (p. 70). Et ce qui s'applique à la traduction vaut tout autant pour l'interprétation. "[...] simultaneous interpreting should be taught by progressing from easy to more difficult, isolating problems and focusing on variables one at a time and, at a later stage, combining them into progressively more intricate structures" (p. 245), écrit Ingrid KURZ dans son article "'Shadowing' Exercises in Interpreter Training". 
3. Enfin, à la lecture de Teaching Translation and Interpreting on ne manque pas de remarquer que les pédagogues et les théoriciens prennent leur distance par rapport à la linguistique (ses méthodes et ses concepts). L'analyse du discours ou, de façon plus générale, les modèles qui prennent en compte la totalité du texte gagnent leur faveur. Gabriella MAURIELLO l'affirme explicitement: "At our school [Civica Scuola Superiore per Interpreti e Traduttori (Milan)], we have based our approach on text linguistics. [...] we tend to treat the text as a whole [...]» (p. 63). C'est le cas aussi dans plusieurs autres écoles de traduction. À maintes reprises, les auteurs citent les travaux des théoriciens Hotz-Mänttäri, Reiss \& Vermeer, Christiane Nord, Mary Snell-Hornby, Basil Hatim \& Ian Mason. La "scopos theory" semble avoir le vent dans les voiles. Mary SnellHornby constate dans la communication: "The study of translation gained real impetus in the early 1980s, when the focus was shifted from the sentence to the text, from the language system to language use [...]" (p. 21). La réflexion sur la traduction semble enfin engagée sur la bonne voie.

Au-delà de ces trois grandes tendances, ce recueil de communications renseigne sur la situation de l'enseignement de la traduction dans le sultanat de Brunei (B. D. SMITH), en Afrique du Sud (Anne-Marie BEUKES) et au Canada (Brian HARRIS). Dans ces trois pays, la traduction a partie liée avec la politique. Est aussi traité l'enseignement de la traduction technique (Gustav WINKLER), du sous-titrage (Henrik GOTTLIEB) et de l'interprétation consécutive (Kambiz MAHMOODZADEH). Comme on le voit, cet ouvrage couvre une grande variété de sujets. Nous n'en avons donné qu'un bref aperçu. Nous attendons avec impatience les actes du deuxième congrès sur ce même thème de l'enseignement. Ce congrès eut lieu du 4 au 6 juin 1993. J'ai été heureux de lire dans The Jerome Quarterly VIII-3, p. 13 sous la plume de David et Margareta Bowen que "Cay Dollerup will again be in charge of the proceedings". Mon petit doigt me dit que nous pourrons lire ces actes au plus tard en juin 1994.

Jean Delisle Université d'Ottawa 